ROCKY MOUNTAIN

JOURNAL OF MATHEMATICS

Volume 24, Number 1, Winter 1994

\title{
OSCILLATION AND ATTRACTIVITY IN \\ A DIFFERENTIAL EQUATION WITH PIECEWISE CONSTANT ARGUMENTS
}

\author{
I.W. RODRIGUES
}

Dedicated to Paul Waltman on the occasion of his 60th birthday

ABSTRACT. Let [-] denote the greatest integer function. Consider the equation with piecewise constant arguments

$$
(*) \quad N^{\prime}(t)=r N(t)\left(1-\sum_{j=0}^{m}\left(a_{j} N\left(\left[t-k_{j}\right]\right)+b_{j} N^{2}\left(\left[t-k_{j}\right]\right)\right)\right)
$$

where $r$ is a positive number and for $j \in\{0,1, \ldots, m\}, k_{j}$ is a nonnegative integer and $a_{j}$ and $b_{j}$ are nonnegative real numbers. We obtain necessary and sufficient conditions for all positive solutions of $(*)$ to oscillate about its positive equilibrium, we provide sufficient conditions for the positive equilibrium of $(*)$ to be a global attractor of all positive solutions, and we establish that every positive solution of $(*)$ is bounded from above and from below by positive constants.

1. Introduction and preliminaries. Let [-] denote the greatest integer function and let $\mathbf{R}$ denote the set of real numbers. Throughout this paper, unless otherwise specified, we will assume that $r \in(0, \infty)$ and for each $j \in\{0,1, \ldots, m\}, k_{j}$ is a nonnegative integer and $a_{j}$ and $b_{j}$ are nonnegative real numbers with $a_{j}+b_{j}>0$. When $k_{j}=0$ for all $j$, we also assume $r \neq 1$.

In this paper we establish necessary and sufficient conditions for the oscillation of all positive solutions of the equation with piecewise constant arguments

$$
N^{\prime}(t)=r N(t)\left(1-\sum_{j=0}^{m}\left(a_{j} N\left(\left[t-k_{j}\right]\right)+b_{j} N^{2}\left(\left[t-k_{j}\right]\right)\right)\right)
$$

about its positive equilibrium. We also obtain sufficient conditions for the positive equilibrium to be a global attractor of all positive solutions.

Received by the editors on March $9,1993$.

AMS 1980 Subject Classification. Primary 39A12, Secondary 39A10.

Copyright (C)1994 Rocky Mountain Mathematics Consortium 
Our motivation to study (1) came from recent studies on equations with piecewise constant arguments. See, for example, $[\mathbf{1}, \mathbf{2}, \mathbf{4}, \mathbf{5}, \mathbf{6}]$.

Throughout this paper, $k$ denotes the maximum of the delays $k_{0}, k_{1}, \ldots, k_{m}$.

By a solution of Equation (1) we mean a continuous function $N(t)$ which is defined on the set $\{-k,-k+1, \ldots, 0\} \cup(0, \infty)$ and which satisfies the following two conditions:

(i) The derivative $N^{\prime}(t)$ exists at each $t \in[0, \infty)$ with the possible exception of the points $t \in\{0,1, \ldots\}$ where one sided derivatives exist and are finite.

(ii) $N(t)$ satisfies Equation (1) in each interval of the form $[n, n+1)$ where $n=0,1, \ldots$.

A solution $N(t)$ of $(1)$ is said to oscillate about a real number $N^{*}$ if the function $N(t)-N^{*}$ has arbitrarily large zeros. Otherwise, the solution is called nonoscillatory about $N^{*}$.

Equation (1) has a unique positive equilibrium. If we denote this equilibrium by $N^{*}$, then

$$
\sum_{j=0}^{m}\left(a_{j} N^{*}+b_{j}\left(N^{*}\right)^{2}\right)=1 .
$$

The following result which is due to Gyori and Ladas [4] is used in the proof of Theorem 1 .

Lemma 1. Consider the nonlinear differential equation with piecewise constant arguments

$$
x^{\prime}(t)+\sum_{j=0}^{m} p_{j} f_{j}\left(x\left(\left[t-k_{j}\right]\right)\right)=0, \quad t \geq 0
$$

where for $j=0,1, \ldots, m$ the following conditions hold:

(a) $p_{j}>0, k_{j}$ is a nonnegative integer and $\sum_{j=0}^{m}\left(p_{j}+k_{j}\right) \neq 1$;

(b) $f_{j} \in C[\mathbf{R}, \mathbf{R}], u f_{j}(u)>0$ for $u \neq 0$ and $\lim _{u \rightarrow 0} f_{j}(u) / u=1$;

(c) there exists a positive number $\delta$ such that for all $j=0,1, \ldots, m$

$$
\text { either } f_{j}(u) \leq u \quad \text { for } 0 \leq u \leq \delta
$$




$$
\text { or } f_{j}(u) \geq u \quad \text { for }-\delta \leq u \leq 0 .
$$

Then every solution of (3) oscillates about zero if and only if

$$
\lambda-1+\sum_{j=0}^{m} p_{j} \lambda^{-k_{j}}=0
$$

has no roots in $(0,1)$.

2. Necessary and sufficient conditions for oscillation. The main goal in this section is to provide necessary and sufficient conditions for the oscillation of all positive solutions of Equation (1) about its positive equilibrium $N^{*}$.

To simplify the notation, we introduce the functions

$$
g_{j}(u)=b_{j} u^{2}+a_{j} u-\left(a_{j} N^{*}+b_{j}\left(N^{*}\right)^{2}\right) \quad \text { for } j=0,1, \ldots, m .
$$

The following lemma gives a useful expression for the solutions of (1).

Lemma 2. Let $N_{0}>0$ and $N_{-j} \in \mathbf{R}$ for $j=1,2, \ldots, k$. Then (1) has a unique positive solution $N(t)$ which is given by

$$
\begin{gathered}
N(t)=N_{n} \exp \left(-r \sum_{j=0}^{m} g_{j}\left(N_{n-k_{j}}\right)(t-n)\right) \\
\text { for } n \leq t<n+1 \quad \text { and } n=0,1, \ldots
\end{gathered}
$$

where the sequence $\left\{N_{n}\right\}$ satisfies the difference equation

$$
N_{n+1}=N_{n} \exp \left(-r \sum_{j=0}^{m} g_{j}\left(N_{n-k_{j}}\right)\right), \quad n=0,1, \ldots
$$

Proof. Set $N_{n}=N(n)$ for $n=-k,-k+1, \ldots$ Then for $t \in[n, n+1)$, (1) becomes

$$
\begin{aligned}
N^{\prime}(t) & =r N(t)\left(1-\sum_{j=0}^{m}\left(a_{j} N_{n-k_{j}}+b_{j} N_{n-k_{j}}^{2}\right)\right) \\
& =-r N(t) \sum_{j=0}^{m} g_{j}\left(N_{n-k_{j}}\right) .
\end{aligned}
$$


By integrating Equation (7) from $n$ to $t$ we obtain (5). By taking limits as $t \rightarrow n+1$ on both sides of (5) and by invoking the continuity of $N(t)$ we obtain (6). The proof is complete.

Let $N(t)$ be a positive solution of Equation (1). Set

$$
N(t)=N^{*} e^{x(t)} \quad \text { for } t \geq 0 .
$$

Observe that $N(t)$ oscillates about $N^{*}$ if and only if $x(t)$ oscillates about zero. Also $x(t)$ satisfies

$$
x^{\prime}(t)=-r \sum_{j=0}^{m} g_{j}\left(N^{*} e^{x\left(\left[t-k_{j}\right]\right)}\right)
$$

or

$$
x^{\prime}(t)+\sum_{j=0}^{m} p_{j} f_{j}\left(x\left(\left[t-k_{j}\right]\right)\right)=0
$$

where

$$
p_{j}=r N^{*}\left(2 b_{j} N^{*}+a_{j}\right) \quad \text { and } \quad f_{j}(u)=\frac{g_{j}\left(N^{*} e^{u}\right)}{2 b_{j}\left(N^{*}\right)^{2}+a_{j} N^{*}} .
$$

We claim that each $f_{j}$ satisfies the hypotheses of Lemma 1 . In fact, if $0<u$ then $N^{*} e^{u}>N^{*}$ and $f_{j}(u)>0$. Similarly, if $u<0$ then $0<N^{*} e^{u}<N^{*}$ and $f_{j}(u)<0$. Therefore $u f_{j}(u)>0$ for $u \neq 0$. Also $f_{j}^{\prime}(0)=1$. Hence the condition (b) of Lemma 1 is satisfied. By using the Mean Value Theorem we will now show that $f_{j}(u)>u$ for $u<0$. Indeed, for $u<0$, there exists $\xi \in(u, 0)$ such that

$$
f_{j}(u)=\frac{g_{j}\left(N^{*} e^{u}\right)-g_{j}\left(N^{*} e^{0}\right)}{2 b_{j}\left(N^{*}\right)^{2}+a_{j} N^{*}}=\frac{2 b_{j}\left(N^{*} e^{\xi}\right)^{2}+a_{j}\left(N^{*} e^{\xi}\right)}{2 b_{j}\left(N^{*}\right)^{2}+a_{j} N^{*}}(u-0) .
$$

Hence $f_{j}(u)>u$. Since $f_{j}(0)=0$, the (c) of Lemma 1 is also satisfied.

Now the following result is obtained by applying Lemma 1 to (9).

Theorem 1. Every positive solution of Equation (1) oscillates about the positive equilibrium $N^{*}$ if and only if the equation

$$
\lambda-1+r N^{*} \sum_{j=0}^{m}\left(2 b_{j} N^{*}+a_{j}\right) \lambda^{-k_{j}}=0
$$


has no roots in $(0,1)$.

Note that if $m=0$, the condition $a_{0} \geq 0$ is not needed. We claim that $f_{0}$ as defined by $(10)$ satisfies all the conditions of Lemma 1 . In fact, it follows from the definition of $N^{*}$ that $p_{0}>0$. Therefore, when $m=0$, Theorem 1 is restated as follows.

Theorem 1'. Assume $r>0, b \geq 0, a \in \mathbf{R}$ which is assumed positive if $b=0$, and let $k$ be a nonnegative integer. If $k=0$ we also assume that $r \neq 1$. Then every positive solution of

$$
N^{\prime}(t)=r N(t)\left(1-a N([t-k])-b N^{2}([t-k])\right), \quad t \geq 0
$$

oscillates about $N^{*}$ if and only if one of the following conditions holds:

$$
r N^{*}\left(2 b N^{*}+a\right)>\frac{k^{k}}{(k+1)^{k+1}} \quad \text { if } k>0
$$

or

$$
r N^{*}\left(2 b N^{*}+a\right)>1 \quad \text { if } k=0 .
$$

If $b=0$ then $N^{*}=1 / a$; otherwise $N^{*}=\left(-a+\sqrt{a^{2}+4 b}\right) / 2 b$.

The following two results are corollaries of Theorem 1.

Corollary 1. Assume $r>0, b>0, a \geq 0$, and let $k$ and $l$ be two positive integers. Let $N_{0}>0$ and $N_{-j} \geq 0$ for $j=1,2, \ldots, \max \{k, l\}$ be given. Then the unique positive solution of

$$
N^{\prime}(t)=r N(t)\left(1-a N([t-k])-b N^{2}([t-l])\right), \quad t \geq 0
$$

oscillates about $N^{*}=\left(-a+\sqrt{a^{2}+4 b}\right) / 2 b$ if and only if

$$
\lambda-1+\operatorname{ar} N^{*} \lambda^{-k}+2 b r\left(N^{*}\right)^{2} \lambda^{-l}=0
$$

has no roots in $(0,1)$.

Corollary 2. If $r \geq 1$ then every positive solution of (1) oscillates about its positive equilibrium $N^{*}$. 
Proof. In view of Theorem 1, it suffices to show that

$$
p(\lambda)=\lambda-1+r N^{*} \sum_{j=0}^{m}\left(2 b_{j} N^{*}+a_{j}\right) \lambda^{-k_{j}}
$$

has no roots in $(0,1)$. If $\lambda \in(0,1)$ it follows from the facts that $r \geq 1$ and $\lambda^{-k_{j}} \geq 1$ and from (2) that

$$
p(\lambda) \geq \lambda-1+N^{*} \sum_{j=0}^{m}\left(2 b_{j} N^{*}+a_{j}\right)=\lambda+\sum_{j=0}^{m} b_{j}\left(N^{*}\right)^{2}>0
$$

Remarks. Theorem $1^{\prime}$ was obtained by Vlahos in [6]. The result of Theorem 1 , under the assumption that $b_{j}=0$ for all $j$, was obtained by Gopalsamy, Kulenovic and Ladas in [2].

3. Global attractivity and boundedness. In this section we establish that all positive solutions of (1) are bounded from above and from below by positive constants, and we obtain sufficient conditions for all positive solutions of (1) to be attracted to the positive equilibrium $N^{*}$.

Theorem 2. (a) Every positive solution of (1) is bounded from above and from below by positive constants.

(b) Assume

$$
N^{*} e^{r(k+1)} \sum_{j=0}^{m}\left(b_{j} N^{*} e^{r(k+1)}+a_{j}\right)<2 .
$$

Then the positive equilibrium $N^{*}$ of (1) is a global attractor of all positive solutions.

Proof. Without loss of generality, we may assume $N_{-j}>0$ for $j=0,1, \ldots, k$. In view of the transformation

$$
N(t)=N^{*} e^{x(t)}
$$


to prove (a), it suffices to show that the solution $x(t)$ of $(8)$ which corresponds to the initial conditions

$$
x_{-j}=\ln \left(\frac{N_{-j}}{N^{*}}\right) \quad \text { for } j=0,1, \ldots, k
$$

is bounded, and to prove (b) it suffices to show that it satisfies

$$
\lim _{t \rightarrow \infty} x(t)=0
$$

First, we define

$$
g(u)=\sum_{j=0}^{m} g_{j}\left(N^{*} e^{u}\right) \quad \text { for } u \in \mathbf{R}
$$

and

$$
M=r(k+1) .
$$

We first show that (12) is satisfied if $x(t)$ is an eventually nonnegative solution of $(8)$. The proof when $x(t)$ is eventually nonpositive is similar and will be omitted.

Note that for each $j=0,1, \ldots, k, N^{*} e^{x\left(\left[t-k_{j}\right]\right)} \geq N^{*}$ eventually so that eventually $g_{j}\left(N^{*} e^{x\left(\left[t-k_{j}\right]\right)}\right) \geq 0$. It follows from $(8)$ that $x^{\prime}(t) \leq 0$ for $n \leq t<n+1$ where $n$ is sufficiently large, say $n \geq n_{0}$. Then

$$
\lim _{t \rightarrow \infty} x(t)=l \geq 0
$$

We claim that $l=0$. Otherwise $l>0$ and by computing the limit as $t \rightarrow \infty$ on both sides of Equation (8), we see that

$$
\lim _{t \rightarrow \infty} x^{\prime}(t)=-r g(l)<0 \text {. }
$$

Hence

$$
x^{\prime}(t)+r g(l) \leq 0 \quad \text { for } n_{0} \leq n \leq t<n+1 .
$$

By integrating both sides of the above inequality from $n$ to $t$ and then by letting $t$ approach $n+1$ we obtain

$$
x(n+1)-x(n)+r g(l) \leq 0 \quad \text { for } n \geq n_{0}
$$


which is clearly impossible for large values of $n$. Therefore, every positive solution of (1) which is eventually in $\left[N^{*}, \infty\right)$ or eventually in $\left(0, N^{*}\right]$ is attracted to $N^{*}$, and, hence, it is bounded. To complete the proof of (a) it remains to show that $x(t)$ is bounded when $x(t)$ is not eventually nonnegative nor eventually nonpositive. In such a situation, there exists a sequence of points $\left\{\xi_{n}\right\}$ satisfying the following properties:

(a) $\lim _{n \rightarrow \infty} \xi_{n}=\infty$;

(b) $k<\xi_{n}<\xi_{n+1}$ and $x\left(\xi_{n}\right)=0$ for $n=1,2, \ldots$;

(c) $x(t)$ assumes both positive and negative values in each interval $\left(\xi_{n}, \xi_{n+1}\right)$.

Let $t_{n}$ and $s_{n}$ be points in $\left(\xi_{n}, \xi_{n+1}\right)$ such that for $n=1,2, \ldots$,

$$
x\left(t_{n}\right)=\max x(t) \quad \text { and } \quad x\left(s_{n}\right)=\min x(t) \quad \text { for } t \in\left(\xi_{n}, \xi_{n+1}\right) .
$$

Then for $n=1,2, \ldots$,

(14)

$$
x\left(t_{n}\right)>0, \quad D^{-} x\left(t_{n}\right) \geq 0, \quad x\left(s_{n}\right)<0 \quad \text { and } \quad D^{-} x\left(s_{n}\right) \leq 0
$$

where $D^{-} x$ denotes the left hand side derivative of $x$.

We now claim that, for each natural number $n$,

$$
x\left(T_{n}\right)=0 \quad \text { for some } T_{n} \in\left[t_{n}-k-1, t_{n}\right)
$$

and

$$
x\left(S_{n}\right)=0 \quad \text { for some } S_{n} \in\left[s_{n}-k-1, s_{n}\right) .
$$

We prove (15). The proof of (16) is handled similarly. Assume, for the sake of contradiction, that (15) does not hold. Since

$$
t_{n} \geq\left[t_{n}-k_{j}\right] \geq\left[t_{n}-k\right] \geq t_{n}-k-1
$$

and $x(t)$ is positive in $\left[t_{n}-k-1, t_{n}\right)$ we see that $g_{j}\left(N^{*} e^{x\left(\left[t_{n}-k_{j}\right]\right)}\right)>0$ for $j=0,1, \ldots, k$. Hence

$$
D^{-} x\left(t_{n}\right)=-r \sum_{j=0}^{m} g_{j}\left(N^{*} e^{x\left(\left[t_{n}-k_{j}\right]\right)}\right)<0
$$


contradicting (14).

By integrating both sides of (8) from $T_{n}$ to $t_{n}$ and by using that each $g_{j}$ is an increasing continuous function on $[0, \infty)$ with the fact that $t_{n}-T_{n} \leq k+1$, we find that for $n=1,2, \ldots$,

$$
\begin{aligned}
x\left(t_{n}\right) & =-r \int_{T_{n}}^{t_{n}} \sum_{j=0}^{m} g_{j}\left(N^{*} e^{x\left(\left[t-k_{j}\right]\right)}\right) d t \\
& \leq-r \sum_{j=0}^{m} g_{j}(0)\left(t_{n}-T_{n}\right) \\
& \leq-r \sum_{j=0}^{m} g_{j}(0)(k+1)=M
\end{aligned}
$$

and so

$$
x(t) \leq M \quad \text { for } t \geq \xi_{1} .
$$

We now use this upper bound to obtain a lower bound for $x(t)$. By integrating both sides of (8) from $S_{n}$ to $s_{n}$ and by using the facts that for each $j=0,1, \ldots, m, g_{j}$ is an increasing function on $[0, \infty)$ and that $0<s_{n}-S_{n} \leq K+1$, we obtain

$$
\begin{aligned}
x\left(s_{n}\right) & =-r \int_{S_{n}}^{s_{n}} \sum_{j=0}^{m} g_{j}\left(N^{*} e^{x\left(\left[t-k_{j}\right]\right)}\right) d t \\
& \geq-r \int_{S_{n}}^{s_{n}} \sum_{j=0}^{m} g_{j}\left(N^{*} e^{M}\right) d t \\
& =-r g(M)\left(s_{n}-S_{n}\right) \geq-M g(M) .
\end{aligned}
$$

We have established that $-M g(M) \leq x(t) \leq M$ for $t \geq \xi_{1}+k+1$. Therefore (a) is proved.

We complete the proof of the theorem by showing that (12) holds when condition (11) is satisfied.

Observe that condition (11) is equivalent to $g(M)<1$, so that

$$
-M \leq x(t) \leq M \quad \text { for } t \geq \xi_{1}+k+1 .
$$

Repeating the above argument with the bounds given in (17) and the fact that $g$ satisfies $g(u) \geq-g(-u)$ for $u \in \mathbf{R}$, we obtain

$$
-M g(M) \leq x(t) \leq M g(M), \quad t \geq \xi_{1}+3(k+1) .
$$


In fact, one can prove by induction that for $n=0,1, \ldots$,

$$
L_{n} \leq x(t) \leq U_{n} \quad \text { for } t \geq \xi_{1}+(2 n+1)(k+1)
$$

where $U_{0}=M$ and

$$
L_{n}=-U_{n} \quad \text { and } \quad U_{n+1}=M g\left(U_{n}\right) .
$$

Moreover,

$$
L_{n} \leq L_{n+1}<0<U_{n+1} \leq U_{n} \quad \text { for } n=0,1, \ldots .
$$

Set

$$
L=\lim _{n \rightarrow \infty} L_{n} \quad \text { and } \quad U=\lim _{n \rightarrow \infty} U_{n} .
$$

In view of (19) we observe that $U=-L$ and that $U$ is a nonnegative zero of the function

$$
\phi(x)=M g(x)-x .
$$

Since $\phi^{\prime \prime}$ is a continuous nonnegative function on $\mathbf{R}, \phi(0)=0, \phi(M)<$ 0 and $0 \leq U \leq M$ we conclude that $L=U=0$. In view of (18), we have that $\lim _{t \rightarrow \infty} x(t)=0$. The proof is complete.

Remark 1. If $b_{j}=0$ for $j=0,1, \ldots, m$, then (1) becomes

$$
N^{\prime}(t)=r N(t)\left(1-\sum_{j=0}^{m} a_{j} N\left(\left[t-k_{j}\right]\right)\right), \quad t \geq 0
$$

where each $a_{j}$ is assumed to be a nonnegative number. Condition (11) reduces to

$$
e^{r(k+1)}<2 .
$$

As it was shown in [2], (22) is a sufficient condition for all positive solutions of $(21)$ to be attracted to its positive equilibrium $N^{*}=$ $\left(\sum_{j=0}^{m} a_{j}\right)^{-1}$. Thus, we see that the term

$$
\sum_{j=0}^{m} b_{j}\left(N^{*} e^{r(k+1)}\right)^{2}
$$


in (11) is due to the introduction of the quadratic term in Equation (21).

Acknowledgments. I am grateful to Professors G. Ladas and E.A. Grove for helpful criticism of the ideas and form of this article.

\section{REFERENCES}

1. L.A.V. Carvalho and Kenneth L. Cooke, A nonlinear equation with piecewise continuous argument, Differential Integral Equations 1 (1988), 359-367.

2. K. Gopalsamy, M.R.S. Kulenovic and G. Ladas, On a logistic equation with piecewise constant arguments, Differential Integral Equations 4 (1991), 215-220.

3. K. Gopalsamy and G. Ladas, On the oscillation and asymptotic behavior of $N^{\prime}(t)=N(t)\left[a+b N(t-\tau)-c N^{2}(t-\tau)\right]$, Quart. Appl. Math. 48 (1990), 433-440.

4. I. Gyori and G. Ladas, Linearized oscillations for equations with piecewise constant arguments, Differential Integral Equations 2 (1989), 123-131.

5. Y.K. Huang, A nonlinear equation with piecewise constant arguments, Appl. Anal. 33 (1989), 183-190.

6. P.N. Vlahos, Oscillation and stability of an equation with piecewise constant quadratic nonlinearity, to appear.

Department of Mathematics, Cleveland State University, Cleveland, Оніо 44115 